\title{
On the Cayley Isomorphism Problem for a Digraph with 24 Vertices
}

\author{
Pablo Spiga \\ Università degli Studi di Padova \\ Dipartimento di Matematica Pura ed Applicata, 35131 Via Trieste 63, Padova, Italy
}

Received 9 January, accepted 15 May 2007, published online 19 June 2008

\begin{abstract}
In this paper we are mainly concerned with the Cayley isomorphism problem for groups containing $Q_{8}$. We prove that the group $Q_{8} \times C_{3}$ is not a CI-group with respect to colour ternary relational structures. Further, we prove that the non-nilpotent group $C_{3} \ltimes Q_{8}$ is not a CI-group with respect to graphs.
\end{abstract}

Keywords: Regular subgroup, Cayley isomorphism.

Math. Subj. Class.: 05E20, 05E99

\section{Introduction}

A $k$-ary relational structure $X$ is an ordered pair $(\Omega, E)$, where $E$ is a subset of the set $\Omega^{k}$. A 3-ary relational structure is also referred to as a ternary relational structure. Further, if we assign a colour to each "edge" of $E$, then the resulting structure $X$ is said to be a colour $k$-ary relational structure. Let $X=(\Omega, E)$ be a colour $k$-ary relational structure. We denote by Aut $X$ the permutation group on $\Omega$ defined by $\left\{\sigma \in \operatorname{Sym}(\Omega) \mid e^{\sigma} \in E\right.$ for any $e \in$ $E$ and $e, e^{\sigma}$ have the same colour $\}$.

Let $G$ be a permutation group on $\Omega$ and $X$ be a (colour) $k$-ary relational structure on $\Omega$. We say that $X$ is a Cayley (colour) $k$-ary relational structure on the group $G$ if the right regular representation of $G$ is contained in Aut $X$. We note that in this case there is a natural bijection between $\Omega$ and $G$. Therefore, $X$ is isomorphic to the (colour) $k$-ary relational structure $(G, F)$, for some subset $F$ of $G^{k}$. In particular, without loss of generality, we can assume that the underlying "vertex-set" of a Cayley (colour) $k$-ary relational structure on $G$ is the group $G$ itself.

E-mail address: spiga@math.unipd.it (Pablo Spiga) 
Recall that if $X=(G, E)$ and $X^{\prime}=\left(G, E^{\prime}\right)$ are Cayley (colour) $k$-ary relational structures on $G$, then $X$ and $X^{\prime}$ are said to be Cayley isomorphic if there exists an automorphism of $G$ that takes $E$ to $E^{\prime}$.

The group $G$ is said to be a CI-group with respect to (colour) $k$-ary relational structures if, for all Cayley (colour) $k$-ary relational structures $X$ and $X^{\prime}$ on $G$, the structures $X$ and $X^{\prime}$ are isomorphic if and only if they are Cayley isomorphic.

See [2] for an account of Cayley colour $k$-ary relational structures and CI-groups. We note that if $k=2$, then we get the usual definition of digraph, Cayley graph and CI-group. Furthermore, it is clear that if $G$ is a CI-group with respect to Cayley colour $k$-ary relational structures, then $G$ is CI-group with respect to Cayley $k$-ary relational structures

We recall that $G$ is a CI-group with respect to (colour) $k$-ary relational structures if and only if, for any Cayley (colour) $k$-ary relational structure $X$ on $G$, any two regular subgroups of Aut $X$ isomorphic to $G$ are conjugate in Aut $X$, see [1].

It is fairly interesting to note that, if $k \geq 4$, then the classification of CI-groups with respect to (colour) $k$-ary relational structures was achieved in [5].

Note that the classification of CI-groups with respect to (colour) graphs is a wide open and very interesting problem, see [4] for an overview of the main results.

We point out that the classification of CI-groups with respect to (colour) ternary relational structures is also wide open. We refer to [2] for an account of this problem.

In Theorem 6 , we prove that $\operatorname{SL}(2,3)$ is not a CI-group with respect to graphs. In particular this result gives further restrictions on the structure of a CI-group and it narrows the list of possible CI-groups given in [4]. We note that $\mathrm{SL}(2,3)$ is isomorphic to $C_{3} \ltimes Q_{8}$, where the action of $C_{3}$ on $Q_{8}$ is non-trivial.

Also, in Theorem 8, we prove that $Q_{8} \times C_{3}$ is not a CI-group with respect to colour ternary relational structures. So, this result improves the list of possible CI-groups with respect to colour ternary relational structures given in [2].

It is worth noticing that $Q_{8}$ and $C_{3}$ are CI-groups with respect to colour ternary relational structures. In particular, $Q_{8} \times C_{3}$ is the only example known to the author of this paper, of a non CI-group with respect to colour ternary relational structures that is the direct product of CI-groups with respect to colour ternary relational structures of coprime order. We would like to point out that no example of this behaviour is known for CI-groups with respect to graphs.

\section{The construction}

Let $Q_{H}$ and $Q_{K}$ be isomorphic to $Q_{8}$, with generators $i_{H}, j_{H}$ and $i_{K}, j_{K}$ (respectively). So, $i_{H}^{2}=j_{H}^{2}=\left[i_{H}, j_{H}\right] \in \xi\left(Q_{H}\right)$ and $i_{H}^{4}=1$, and similar relations hold for the group $Q_{K}$. In this paper, $\xi(G)$ denotes the centre of a group $G$.

We denote by $E$ the extraspecial group $Q_{H} \circ Q_{K}$, i.e. the central product of $Q_{H}$ and $Q_{K}$, see [3]. In other words $E$ is the direct product of $Q_{H}$ and $Q_{K}$ with their centres identified, i.e. $E=\left(Q_{H} \times Q_{K}\right) /\left\langle i_{H}^{2} i_{K}^{2}\right\rangle$. Then $E$ is the 2 -group of order 32 with generators $i_{H}, j_{H}, i_{K}, j_{K}$ and with relations

$$
\begin{aligned}
i_{H}^{4}=\left[i_{H}, i_{K}\right]=\left[i_{H}, j_{K}\right]=\left[j_{H}, i_{K}\right]=\left[j_{H}, j_{K}\right]=1, \\
i_{H}^{2}=i_{K}^{2}=j_{H}^{2}=j_{K}^{2}=\left[i_{H}, j_{H}\right]=\left[i_{K}, j_{K}\right] \in \xi(E) .
\end{aligned}
$$

We recall that if $G$ is a finite group and $\varphi: G \rightarrow G$ is a function mapping a generating 
set of $G$ to another generating set of $G$ and preserving the defining relations of $G$, then $\varphi$ is an automorphism of $G$.

Now we denote by $C$ the subgroup of $\operatorname{Aut}(E)$ generated by $x, y, t$, where $x, y$ and $t$ are defined as follows:

$$
\begin{array}{cccc}
i_{H}^{x}=j_{H}, & j_{H}^{x}=i_{H} j_{H}, & i_{K}^{x}=i_{K} j_{K}, & j_{K}^{x}=i_{K} \\
i_{H}^{y}=i_{H} j_{H}, & j_{H}^{y}=i_{H}, & i_{K}^{y}=i_{K} j_{K}, & j_{K}^{y}=i_{K} \\
i_{H}^{t}=i_{K}, & j_{H}^{t}=j_{K}, & i_{K}^{t}=i_{H}, & j_{K}^{t}=j_{H} .
\end{array}
$$

Using the previous paragraph and the relations of $E$ given above, the reader may check that $x, y$ and $t$ define automorphisms of $E$.

Lemma 1. $(i) t^{2}=x^{3}=y^{3}=1$,

(ii) $x^{t}=x^{-1},[x, y]=1$ and $[t, y]=1$ (so $y$ is in the centre of $C$ ),

(iii) $C$ is isomorphic to $\operatorname{Sym}(3) \times C_{3}$, and

(iv) xy centralizes $i_{H}, j_{H}$ and $x y^{-1}$ centralizes $i_{K}, j_{K}$.

Proof. (i) By definition of $t$, we have that $t^{2}$ fixes $i_{H}, j_{H}, i_{K}, j_{K}$. Therefore, $t^{2}$ fixes every element of $E$, thus $t^{2}=1$. Now,

$$
i_{H}^{x^{3}}=\left(i_{H}^{x}\right)^{x^{2}}=\left(j_{H}^{x}\right)^{x}=\left(i_{H} j_{H}\right)^{x}=j_{H} i_{H} j_{H}=i_{H} .
$$

This yields that $x^{3}$ centralizes $i_{H}$. Similarly, the reader can check that $x^{3}, y^{3}$ centralize the generators $i_{H}, j_{H}, i_{K}, j_{K}$ of $E$. Therefore $x^{3}=y^{3}=1$.

(ii) We note that $i_{H}^{x^{t}}=i_{H}^{t x t}=i_{K}^{x t}=\left(i_{K} j_{K}\right)^{t}=i_{H} j_{H}=i_{H}^{x^{-1}}$. Similarly, the reader can check that $j_{H}^{x^{t}}=j_{H}^{x^{-1}}, i_{K}^{x^{t}}=i_{K}^{x^{-1}}, j_{K}^{x^{t}}=j_{K}^{x^{-1}}$. This says that $x^{t}=x^{-1}$. The proofs that $[y, t]=1$ and $[x, y]=1$ are analogous.

(iii) It follows from $(i),(i i)$.

(iv) By definition of $x$ and $y$, we have $i_{H}^{x y}=j_{H}^{y}=i_{H}$ and $j_{H}^{x y}=\left(i_{H} j_{H}\right)^{y}=i_{H} j_{H} i_{H}=$ $j_{H}$. Thus $x y$ centralizes $i_{H}, j_{H}$. Similarly, the reader can check that $x y^{-1}$ centralizes $i_{K}, j_{K}$.

If $H$ is a subgroup of a group $G$, then we say that $H$ is a core-free subgroup of $G$ if the only normal subgroup of $G$ contained in $H$ is 1, i.e. $\cap_{g \in G} H^{g}=1$. Recall that if $H$ is a core-free subgroup of $G$, then the action of $G$ on the right cosets of $H$ in $G$ is faithful.

Now we denote by $A$ the group $C \ltimes E$. Consider $B=\left\langle i_{H} i_{K}^{-1}, t, x\right\rangle$. We denote by $v_{1}$ the element $i_{H} i_{K}^{-1}$, and set $v_{2}=v_{1}^{x}, v_{3}=v_{2}^{x}$ and $B_{E}=B \cap E$.

Lemma 2. The group $B_{E}$ is an elementary abelian 2-group of order 4 and consists of $\mathrm{id}, v_{1}, v_{2}, v_{3}$. The group $B$ is isomorphic to $\operatorname{Sym}(4)$ and $B \cap B^{y}=\langle x, t\rangle$. The group $B$ is a core-free subgroup of $A$.

Proof. The elements $v_{1}, v_{2}, v_{3}$ have order 2 and

$$
v_{1} v_{2}=i_{H} i_{K}^{-1} j_{H}\left(i_{K} j_{K}\right)^{-1}=i_{H} j_{H} j_{K}^{-1}=v_{3} .
$$

So, $v_{1}, v_{2}, v_{1} v_{2}$ are involutions and hence $v_{1}$ and $v_{2}$ commute. Therefore $\left\langle v_{1}, v_{2}, v_{3}\right\rangle$ is an elementary abelian 2-group of order 4 . Further, $v_{1}^{t}=v_{1}$ and $v_{2}^{t}=v_{3}$. This shows that $B_{E}=\left\langle v_{1}, v_{2}\right\rangle$. In particular, $B_{E}=\left\{\mathrm{id}, v_{1}, v_{2}, v_{3}\right\}$. 
Now, $B=\langle x, t\rangle \ltimes B_{E}$ and Lemma $1(i),(i i)$ yields $B \cong \operatorname{Sym}(4)$.

By Lemma $1(i i)$, we have $B \cap B^{y} \geq\langle x, t\rangle$. Now, as $B$ is isomorphic to $\operatorname{Sym}(4)$ and $\langle x, t\rangle$ is isomorphic to $\operatorname{Sym}(3)$, we have that either $B \cap B^{y}=\langle x, t\rangle$ or $B=B^{y}$. Since $v_{1}^{y}=i_{H} j_{H}\left(i_{K} j_{K}\right)^{-1} \in B^{y} \backslash B$, we get $B \cap B^{y}=\langle x, t\rangle$.

Thus, if $B$ contains a normal subgroup $N$ of $A$, we must have $N \leq\langle x, t\rangle$. But $\langle x, t\rangle \cong$ $\operatorname{Sym}(3)$, and the only subgroup of $\operatorname{Sym}(3)$ normal in $\operatorname{Sym}(4)$ is 1 . So $N=1$. Thus $B$ is a core-free subgroup of $A$. $\left.x y^{-1}\right\rangle$.

Define $H=\left\langle i_{H}, j_{H}, x y\right\rangle, K=\left\langle i_{K}, j_{K}, x y^{-1}\right\rangle, U=\left\langle i_{H}, j_{H}, y\right\rangle$ and $V=\left\langle i_{H}, j_{H}\right.$,

Note that, by the definition of $x, y$ and by Lemma $1(i v)$, the groups $H, K$ are isomorphic to $Q_{8} \times C_{3}$ and $U, V$ are isomorphic to $\operatorname{SL}(2,3)$ (we recall that $\operatorname{SL}(2,3)$ is isomorphic to $C_{3} \ltimes Q_{8}$, where the action of $C_{3}$ on $Q_{8}$ is non-trivial).

Lemma 3. $B \cap H=B \cap K=B \cap U=B \cap V=1$ and $B H=B K=B U=B V=A$.

Proof. We first prove that $B \cap H=B \cap K=1$ and $B H=B K=A$. Since $t$ lies in $B$ and $H^{t}=K$, it is enough to prove that $B \cap H=1$ and $B H=A$. We have $B \cap$ $H \subseteq E\langle x, t\rangle \cap E\langle x y\rangle=E$. So, $B \cap H=B \cap(H \cap E)=B \cap\left\langle i_{H}, j_{H}\right\rangle=1$. Since $|A|=|E||C|=32 \cdot 18=24 \cdot 24=|H||B|$ and $B \cap H=1$, we have $B H=H B=A$.

Now, we prove that $B \cap U=1$ and $B U=A$. We have $B \cap U \subseteq E\langle x, t\rangle \cap E\langle y\rangle=E$. So, $B \cap U=B \cap(U \cap E)=B \cap\left\langle i_{H}, j_{H}\right\rangle=1$. Since $|A|=|U||B|$ and $B \cap H=1$, we have $B U=A$. Similarly, the reader can check that $B \cap V=1$ and $B V=A$.

Next, we consider the action of $A$ on the right cosets $\Omega=A / B$. Lemma 2 yields that $A$ is a permutation group of degree 24 with point stabilizer isomorphic to $\operatorname{Sym}(4)$. Lemma 3 yields that $H, K, U$ and $V$ are regular subgroups of $A$.

Lemma 4. Let $\Delta$ be the B-orbit of the point By of $\Omega$. We have

$$
\Delta=\left\{B y, B y v_{1}, B y v_{2}, B y v_{3}\right\}
$$

and the action of $B$ on $\Delta$ is equivalent to the action of $\operatorname{Sym}(4)$ on four points.

Proof. By Lemma 2, we have $B \cap B^{y}=\langle x, t\rangle$. Therefore, the group $B_{E}$ acts regularly on $\Delta$. Since $y$ centralizes $\langle x, t\rangle$, we have that every element of $\langle x, t\rangle$ fixes $B y$. So, $\Delta=$ $\left\{B y, B y v_{1}, B y v_{2}, B y v_{3}\right\}$. Moreover,

$$
B y v_{1} x=B x\left(y v_{1}\right)^{x}=B y v_{2}, B y v_{2} x=B x\left(y v_{2}\right)^{x}=B y v_{3} .
$$

This shows that $x$ acts on $\Delta$ as a 3 -cycle. Similarly,

$$
B y v_{1} t=B t y v_{1}=B y v_{1}, B y v_{2} t=B t\left(y v_{2}\right)^{t}=B y v_{3} .
$$

This says that $t$ acts on $\Delta$ as a 2-cycle. Thus the lemma is proved.

Let $\mathcal{S}$ be the orbital corresponding to the suborbit $\Delta$ of $A$, i.e. $\mathcal{S}=\{(B a, B y a) \mid a \in A\}$. Let $\Gamma$ be the orbital digraph of $A$ corresponding to the orbital $\mathcal{S}$, we recall that $\Gamma$ has vertex set $\Omega$ and edge set $\mathcal{S}$. We note that, by construction, $A$ is a subgroup of Aut $\Gamma$.

Proposition 5. $A=$ Aut $\Gamma$.

Proof. Since $B$ acts 4-transitively on $\Delta$, it is enough to prove that if $\sigma$ is an automorphism of $\Gamma$ fixing the vertex $B$ and the out-neighbours of $B$, then $\sigma=$ id. We leave this routine exercise to the conscientious reader. 


\section{$3 \mathrm{SL}(2,3)$}

In this section, we prove that $\mathrm{SL}(2,3)$ is not a CI-group (with respect to graphs). We recall that $U \cong V \cong \mathrm{SL}(2,3)$.

Theorem 6. The group $\mathrm{SL}(2,3)$ is not a $\mathrm{CI}-$ group with respect to graphs.

Proof. The groups $U, V$ are regular subgroups of $A$ isomorphic to $\operatorname{SL}(2,3)$. Furthermore, by Proposition 5, the group $A$ is the automorphism group of the digraph $\Gamma$. In particular, $\Gamma$ is a Cayley graph on $\mathrm{SL}(2,3)$. Therefore, it is enough to prove that $U, V$ are not conjugate in $A$. We argue by contradiction. Let $g$ be in $A$ such that $U^{g}=V$. Since $\left\langle i_{H}, j_{H}\right\rangle$ is a characteristic subgroup of $U$ and $V$, we have $\left\langle i_{H}, j_{H}\right\rangle^{g}=\left\langle i_{H}, j_{H}\right\rangle$, i.e. $g \in N_{A}\left(\left\langle i_{H}, j_{H}\right\rangle\right)=\langle x, y\rangle E$. Now, $g=z e$ for some $z \in\langle x, y\rangle$ and $e \in E$. The element $y$ lies in $U$, therefore $y^{g}=$ $\left(y^{z}\right)^{e}=y^{e}=y[y, e]$ lies in $V$. But $V \subseteq\left\langle x y^{-1}\right\rangle E$ and $[y, e] \in E$, so $y \in\left\langle x y^{-1}\right\rangle$, a contradiction.

\section{$4 Q_{8} \times C_{3}$}

In this section, we prove that $Q_{8} \times C_{3}$ is not a CI-group with respect to colour ternary relational structures. We recall that $H \cong K \cong Q_{8} \times C_{3}$.

Set $G=E\langle x, y\rangle$. Let $\mathcal{T}_{1}$ be the subset of $\Omega^{3}$ defined by $\{(B g, B g, B y g) \mid g \in G\}$. Also, let $\mathcal{T}_{2}$ be the subset of $\Omega^{3}$ given by $\left\{\left(B g, B i_{H} g, B j_{H}^{-1} g\right) \mid g \in G\right\}$. The sets $\mathcal{T}_{1}, \mathcal{T}_{2}$ define two ternary relational structures on $\Omega$. We recall that Aut $\mathcal{T}_{i}=\left\{\sigma \in \operatorname{Sym}(\Omega) \mid t^{\sigma} \in\right.$ $\mathcal{T}_{i}$ for any $\left.t \in \mathcal{T}_{i}\right\}$, for $i=1,2$.

Proposition 7. $G=\operatorname{Aut} \mathcal{T}_{1} \cap \operatorname{Aut} \mathcal{T}_{2}$.

Proof. We claim that $A=$ Aut $\mathcal{T}_{1}$. The group $G$ is a transitive subgroup of $A$ and, by Lemma 4, the stabilizer in $G$ of the point $B$ of $\Omega$ is isomorphic to Alt(4) and acts transitively on $\Delta$. This says that $\{(B g, B y g) \mid g \in G\}=\{(B a, B y a) \mid a \in A\}=\mathcal{S}$. Let $\sigma$ be in Aut $\mathcal{T}_{1}$ and $e$ be in $\mathcal{S}$. Now, $e=(B g, B y g)$, for some $g \in G$. Set $\tau=(B g, B g, B y g)$. Now, $\tau \in \mathcal{T}_{1}$, therefore $\tau^{\sigma} \in \mathcal{T}_{1}$. So, $\tau^{\sigma}=\left(B g^{\prime}, B g^{\prime}, B y g^{\prime}\right)$, for some $g^{\prime} \in G$. In particular $e^{\sigma}=\left(B g^{\prime}, B y g^{\prime}\right) \in \mathcal{S}$. Since $e$ is an arbitrary element of $\mathcal{S}$, we get $\sigma \in$ Aut $\Gamma=A$. Since $\sigma$ is an arbitrary element of Aut $\mathcal{T}_{1}$, we get Aut $\mathcal{T}_{1} \subseteq A$. By a similar argument, $A \subseteq$ Aut $\mathcal{T}_{1}$. Therefore, $A=$ Aut $\mathcal{T}_{1}$.

By construction, the group $G$ is a subgroup of Aut $\mathcal{T}_{i}$, for $i=1,2$. The group $G$ has index 2 in $A$ and $A=G\langle t\rangle$. Therefore $G=$ Aut $\mathcal{T}_{1} \cap$ Aut $\mathcal{T}_{2}$ if and only if $t \notin$ Aut $\mathcal{T}_{2}$. Now, by Lemma $1,\left(B, B i_{H}, B j_{H}^{-1}\right)^{t}=\left(B t, B i_{H} t, B j_{H}^{-1} t\right)=\left(B, B i_{K}, B j_{K}^{-1}\right)$. Since there is no element $g \in G$ such that $\left(B g, B i_{H} g, B j_{H}^{-1} g\right)=\left(B, B i_{K}, B j_{K}^{-1}\right)$, we have $t \notin$ Aut $\mathcal{T}_{2}$. Thus the proposition is proved.

Theorem 8. The group $Q_{8} \times C_{3}$ is not a CI-group with respect to colour ternary relational structures.

Proof. The groups $H, K$ are regular subgroups of $G$. Furthermore, by Proposition 7, the group $G$ is the automorphism group of a colour ternary relational structure (indeed, with just two colours: $\left.\mathcal{T}_{1}, \mathcal{T}_{2}\right)$. In particular, $\mathcal{T}_{i}$ is a Cayley ternary relational structure on $Q_{8} \times C_{3}$, for $i=1,2$. So, it is enough to prove that $H, K$ are not conjugate in $G$. We argue by contradiction. Let $g$ be in $G$ such that $H^{g}=K$. Clearly, $\left\langle i_{H}, j_{H}\right\rangle^{g}=\left\langle i_{K}, j_{K}\right\rangle$. Since $\left\langle i_{H}, j_{H}\right\rangle$ is a normal subgroup of $G$, we get a contradiction. 


\section{References}

[1] L. Babai, Isomorphism problem for a class of point-symmetric structures, Acta Math. Acad. Sci. Hungar. 29 (1977), 329-336.

[2] E. Dobson, On the Cayley isomorphism problem for ternary relational structures, J. Combin. Theory Ser. A 101 (2003), no. 2, 225-248.

[3] C. R. Leedham-Green and S. McKay, The structure of groups of prime power order, London Mathematical Society Monographs. New Series, 27, Oxford Science Publications, Oxford University Press, Oxford, 2002. xii+334 pp.

[4] C. H. Li, On Isomorphisms of finite Cayley graphs - a survey, Discrete Math. 246 (2002), 301-334.

[5] P. P. Pálfy, Isomorphism problem for relational structures with a cyclic automorphism, European Journal of Comb. 8 (1987), 35-43. 